\title{
On-board Experimental Study of Bubble Thermocapillary Migration in a Recoverable Satellite
}

\author{
Q. Kang $\cdot$ H. L. Cui $\cdot$ L. Hu $\cdot$ L. Duan
}

Received: 30 December 2006 / Accepted: 26 October 2007 / Published online: 28 March 2008

(C) Springer Science + Business Media B.V. 2008

\begin{abstract}
An on-board space experiment of bubble thermocapillary migration was performed in the Chinese 22nd recoverable satellite in 2005. Silicone oil of nominal viscosity $5 \mathrm{cSt}$ was used as the continuous phase in the experiment. Air bubbles were injected into the liquid in the same direction as the constant temperature gradient in the liquid. The velocities of bubbles were obtained by recording the paths of the bubbles. The results indicate that the scaled velocity of bubbles decreases with an increase of the Marangoni number extended to 9288, which agrees with the results of previous space experiments and numerical simulation. In addition, the interaction between two bubbles was also observed in the space experiment. The trajectories and the velocities of the bubbles were obtained. The twobubble experiment results are also consistent with the theoretical analysis.
\end{abstract}

Keywords Space experiment - Thermocapillary migration . Bubble interaction $\cdot$ Microgravity fluid

\section{Introduction}

A bubble or a drop in an immiscible fluid will move toward the warmer side when subjected to a temperature gradient in microgravity environment. Such a phenomenon is known as the Marangoni problem or the thermocapillary migration problem. Bubble and/or drop dynamics has become a hot point of research because this investigation is very important for fundamental research and applications in

Q. Kang $(\bowtie) \cdot$ H. L. Cui $\cdot$ L. Hu $\cdot$ L. Duan

National Microgravity Laboratory/CAS; Institute of Mechanics,

Chinese Academy of Sciences,

Beijing 100080, China

e-mail: kq@imech.ac.cn reduced gravity environment, such as space material science, chemical engineering, and so on. In this paper, we present some results on bubble thermocapillary migration and bubble interaction from the space experiments on the Chinese 22nd recoverable satellite in 2005.

In 1959, Young et al. (1959) first investigated the thermocapillary migration of bubbles and drops with their linear model, which is suitable for small Reynolds number $(R e)$ and small Marangoni number $(M a)$. This model is now called the YGB model. The Reynolds number is the ratio of inertial forces to viscous forces. Small $R e$ means that inertial effects are negligible. The Marangoni number is the ratio of convective transport of energy to heat conduction. Small $M a$ means that convective heat transfer is negligible compared to heat conduction. On the basis of small $R e$ and small $M a$, YGB model velocity could be obtained from the linear assumption. $R e, M a$, reference velocity $V_{0}$, thermal diffusivity $K$ and YGB model velocity $V_{\text {YGB }}$ (Young et al. 1959) are expressed as follows:

$\operatorname{Re}=\frac{R V_{0}}{v}$

$M a=\frac{R V_{0}}{\kappa}$

$V_{0}=\frac{\left|\sigma_{T}\right||\nabla T| R}{\mu}$

$\kappa=\frac{\lambda}{\rho c_{p}}$

$V_{Y G B}=\frac{2\left|\sigma_{t}\right| R \lambda \nabla T}{\left(2 \mu+3 \mu^{\prime}\right)\left(2 \lambda+\lambda^{\prime}\right)}$ 
Where $R$ is the radius of the bubble, $\nabla T$ is the temperature gradient, $\sigma_{T}$ is the rate of change of surface tension with temperature, $\mu$ and $\mu^{\prime}, \lambda$ and $\lambda^{\prime}$ are the dynamic viscosity, thermal conductivity of continuous phase and air, respectively. $v$ and $c_{\mathrm{p}}$ are the kinematic viscosity and the specific heat of continuous phase. $\rho$ and $\kappa$ are the density and thermal diffusivity of continuous phase. For air bubbles, because the dynamic viscosity and thermal conductivity of the air are much less than that of the continuous phase, the reference velocity, $V_{0}$, is approximately twice the velocity of YGB model, $V_{\mathrm{YGB}}$.

Over the last decades, many researchers have studied the thermocapillary migration of bubbles and drops. Many theoretical and numerical methods have been used to correct the YGB model for large Re and large $M a$ (Subramanian and Balasubramaniam 2001; Subramanian et al. 2001). The experiments of thermocapillary migration of bubbles and drops performed on the ground are limited by the effect of gravity (Subramanian and Balasubramaniam 2001; Subramanian et al. 2001) reported the experiments on thermocapillary migration under microgravity environments performed in the drop tower, rocket and spacecraft. Most of the valid results were attained from the experiments performed in the last three space flights. Two of them were, respectively, the International Microgravity Laboratory Mission (IML-2) in 1994 (Balasubramaniam et al. 1996) and the Life and Microgravity Science (LMS) Mission in 1996 (Hadland et al. 1999) on the Columbia Space Shuttle. The other was on China's ShenZhou-4 spacecraft in 2002, where single-drop thermocapillary migration was studied, and the largest $M a$ (5500) in drop thermocapillary migration was obtained (Xie et al. 2005). In the LMS mission, $R e$ and $M a$ of bubble thermocapillary migration were extended from 0.839 to 87.2 and from 51.7 to 5780 , respectively.

In fact, the interactions between bubbles or drops are more common than migration of a single bubble (Meyyappan et al. 1983) studied the axisymmetric thermocapillary migration of two gas bubbles in the quasi-steady state with bi-spherical coordinates. Afterwards, (Meyyappan and Subramanian 1984) studied the thermocapillary migration of two bubbles in arbitrary angles to the applied temperature gradient with zero-order reflection approximation. Anderson (1985) then extended the reflection solution to the first order and obtained the velocities of two arbitrarily oriented droplets up to terms of $\mathrm{O}\left(r_{12}^{-6}\right)$, where $r_{12}$ is the distance between the two drops. All of these approximate solutions, however, are invalid when the bubbles or drops move close enough. Sun and $\mathrm{Hu}(2002,2003)$ used the successive reflection technique to obtain the analytical solution shown in Fig. 1. The results indicate that the interaction between two bubbles has a significant influence on the migration of the smaller bubble. When the larger bubble approaches the smaller one, the smaller one's speed may
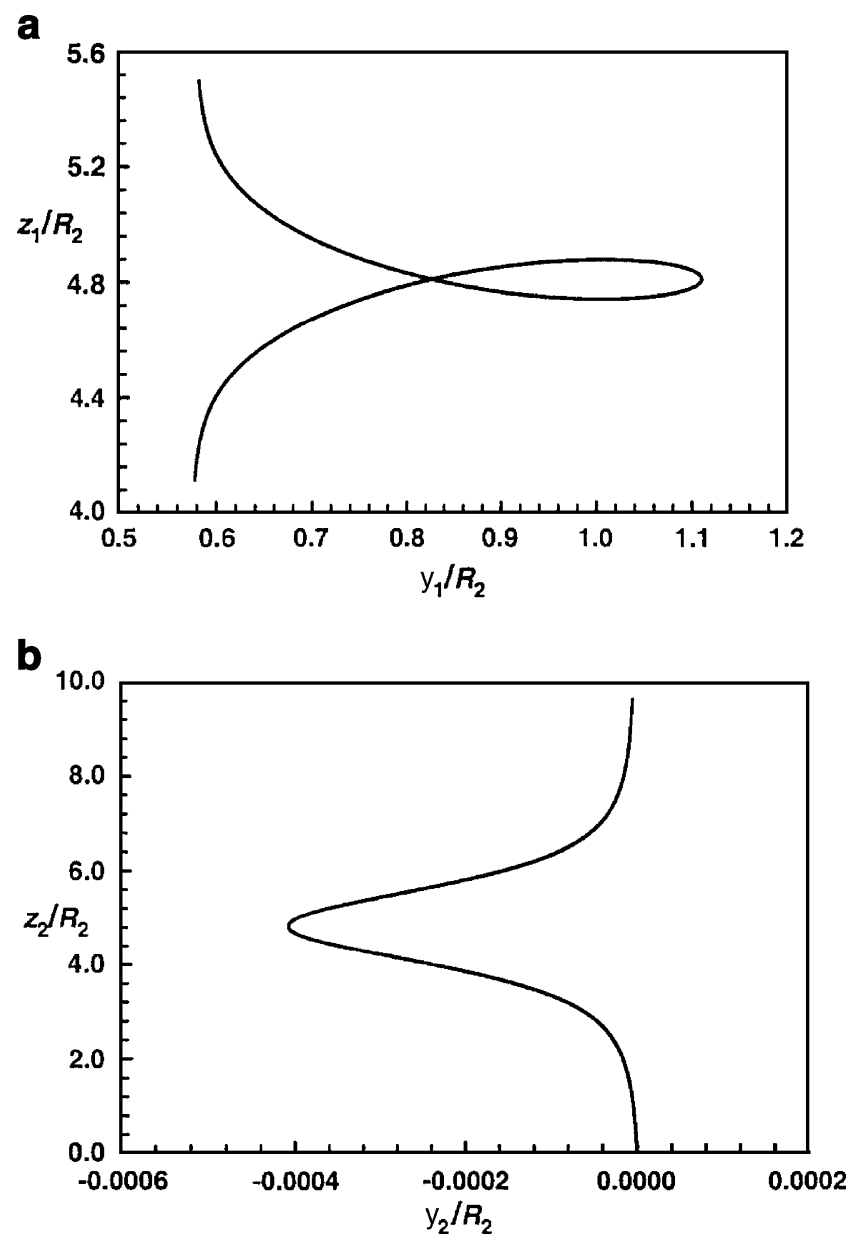

Fig. 1 Motions of two bubbles. a Smaller bubble's trajectories. b Larger bubble's trajectories

decrease to zero and even move backwards, and the larger bubble drives the smaller one aside. All the theoretical work about interactions between bubbles or drops mentioned above was restricted to small Re and $M a$. Recently, numerical simulations of the thermocapillary interaction between three-dimensional fully deformable bubbles or drops at large $R e$ and $M a$ were reported by Nas and Tryggvason (2003) and Nas et al. (2006), whose results show that bubbles and drops tend to line up perpendicular to the direction of temperature gradient, which contrasts with results at small $R e$ and $M a$.

\section{Experimental Facility}

As shown in Fig. 2, the rectangular test chamber was $70 \mathrm{~mm}$ high and $40 \times 40 \mathrm{~mm}^{2}$ in horizontal cross section. The experimental cell was composed of four optical glass walls with two aluminum plates at the top and on the bottom. A piece of electric heating film was placed on the top above the aluminum plate and a Peltier element on the bottom 
Fig. 2 Schematic of the experimental apparatus

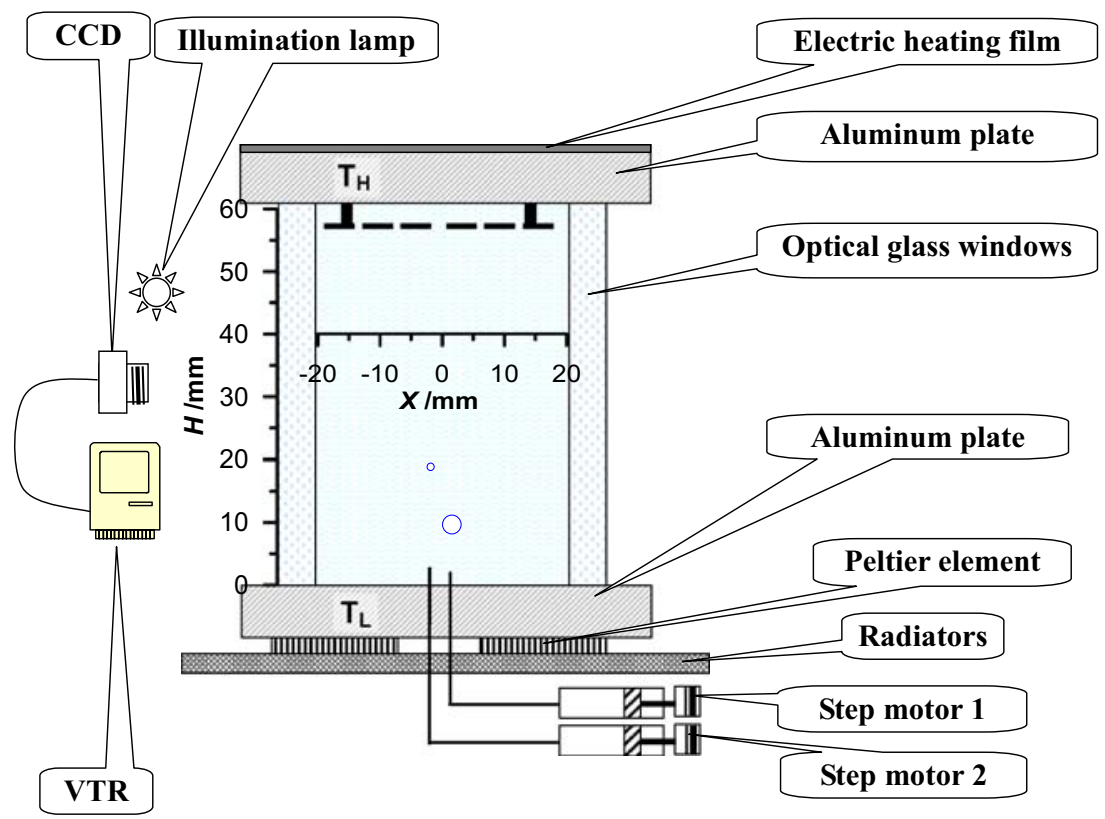

under the aluminum plate. A grid plate was fixed near the top to ensure uniformity of the temperature distribution. In order to maintain a steady temperature difference between the top and the bottom aluminum plates, two PID temperature controllers were used to maintain constant temperatures of the plates. Two small-diameter cylinders (made by FESTO GmbH) and two Stepper-Mike Actuators (made by Physik Instrument $\mathrm{GmbH} \& \mathrm{Co}$ ) are used to control the size of the bubbles injected. There is a distance of $3 \mathrm{~mm}$ between the two bubble injecting holes on the bottom, which are for the research of the interaction between the un-coaxial bubbles. The temperature field of continuous phase in the test cell was measured with six Ktype thermocouples, which were placed on the side wall, from the top down. The migration path of the bubble was recorded by VTR. The recording format of video image is PAL with a video rate of 25 frames per second. The time resolution was $0.04 \mathrm{~s}$ and the space resolution of the image was $0.1 \mathrm{~mm}$ per pixel. The recorded temperature data and video image of bubble migration in the experiment, together with the test facility were returned along with the recoverable cabin of the satellite. The peak acceleration level aboard the satellite during the experiment was less than $10^{-3} g_{0}$ from the monitored data, where $g_{0}$ is the acceleration of gravity at the surface of the earth, so the influence from the buoyancy force could be ignored.

\section{Results of Space Experiment}

5cSt silicone oil produced by Shin-Etsu Chemical Co., Ltd. was used as the continuous phase in the space experiment. The physical property parameters of the $5 \mathrm{cSt}$ silicone oil come from Xie et al. (2005). The temperature controllers were started 100 min earlier than the air stored in the cylinder was injected as the dispersed phase in order to get a steady temperature field. At the start time when the bubbles were injected, the measured temperature gradient in the middle of the test cell was $\nabla T=0.73\left({ }^{\circ} \mathrm{C} / \mathrm{mm}\right)$. The size of the air bubbles and the time interval between injections were controlled by the preconcerted program. More than ten groups of single-bubble experiments and six groups of two or three-bubble experiments of the thermocapillary migration and interaction of bubbles were completed in the space experiment. The whole space experiment lasted $220 \mathrm{~min}$.

The bubble's migration velocity was obtained by measuring the bubble's tracks. All the bubbles migrated near the center axis of the cell. The depth of field of the camera was long enough to capture bubble images clearly. The uncertainty limits of the smallest velocity $\delta V$ and the largest velocity $V_{\max }$ are $0.05 \mathrm{~mm} / \mathrm{s}$ and $1.5 \mathrm{~mm} / \mathrm{s}$, respectively. In the experiment of bubble thermocapillary migration, Re and $M a$ range from 2.3 to 217.9 and from 98.04 to 9288 , respectively. The maximum $M a$ of bubble thermocapillary migration reached 9288 , which is larger than that attained in any prior space experiment. In the case of the maximum $M a$ in the experiment, the scaled migration velocity $V / V_{\mathrm{YGB}}$ is 0.4 . The results show that the migration velocity of bubbles, in this case, is smaller than that in the YGB model. On the other hand, the migration velocity also increases with an increase of the bubble size as in the YGB model. The curve of the scaled velocity versus $M a$ is shown in Fig. 3, which is similar to the result predicted by Balasubramaniam and Subramanian (1996); 


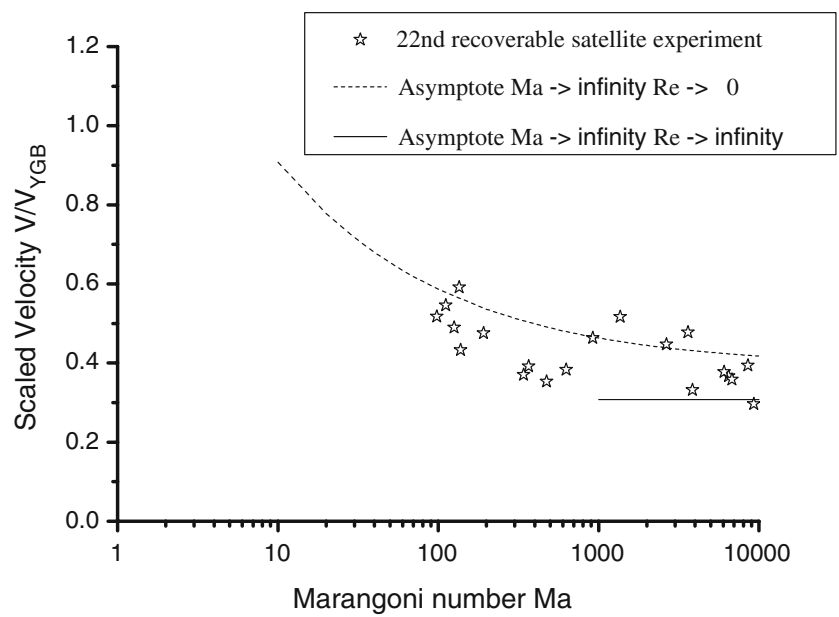

Fig. 3 Scaled velocity versus $M a$

Ma et al. (1999) as shown in Eqs. (6) and (7). The comparisons between the experimental results and theoretical analyses demonstrate similarities and differences.

As $\mathrm{Ma} \rightarrow \infty, \quad \frac{V}{V_{Y G B}}=0.3076, \operatorname{Re} \rightarrow 0$
$\frac{V}{V_{Y G B}}=0.3920+\frac{0.1369}{\sqrt{M a}}[9.610+\operatorname{In}(M a)], \operatorname{Re} \rightarrow \infty$

In this experiment, the chase and interaction between uncoaxial bubbles are observed. The track and velocities of bubbles in two cases of different radius ratios are shown in Fig. $4 \mathrm{a}$ and $\mathrm{b}$. The scaled initial distances $\left(H_{0} / R_{1}\right)$ between axes in Fig. $4 \mathrm{a}$ and $\mathrm{b}$ are 4.76 and 5.04, respectively. The attenuations of small bubbles due to interaction between bubbles were obvious. If the size ratio of the bubbles is large, the smaller one will come close to rest in a short period, while the two bubbles migrate side by side. This phenomenon is similar in tendency to the theoretical prediction at small $\mathrm{Ma}$ and small $\mathrm{Re}$ (Sun and $\mathrm{Hu}$ 2002, 2003) as shown in Fig. 1. According to the velocity curves in Fig. 4, the effective range of interaction during chase and surpass is $0.5-1.1$ times of larger bubble's diameter. However, according to the results from Meyyappan's linear theory (Meyyappan et al. 1983), when the distance between two bubbles is $0.8-1.4$ times the larger bubble's diameter, the relative change of the small bubble's velocity will reach $20 \%$.
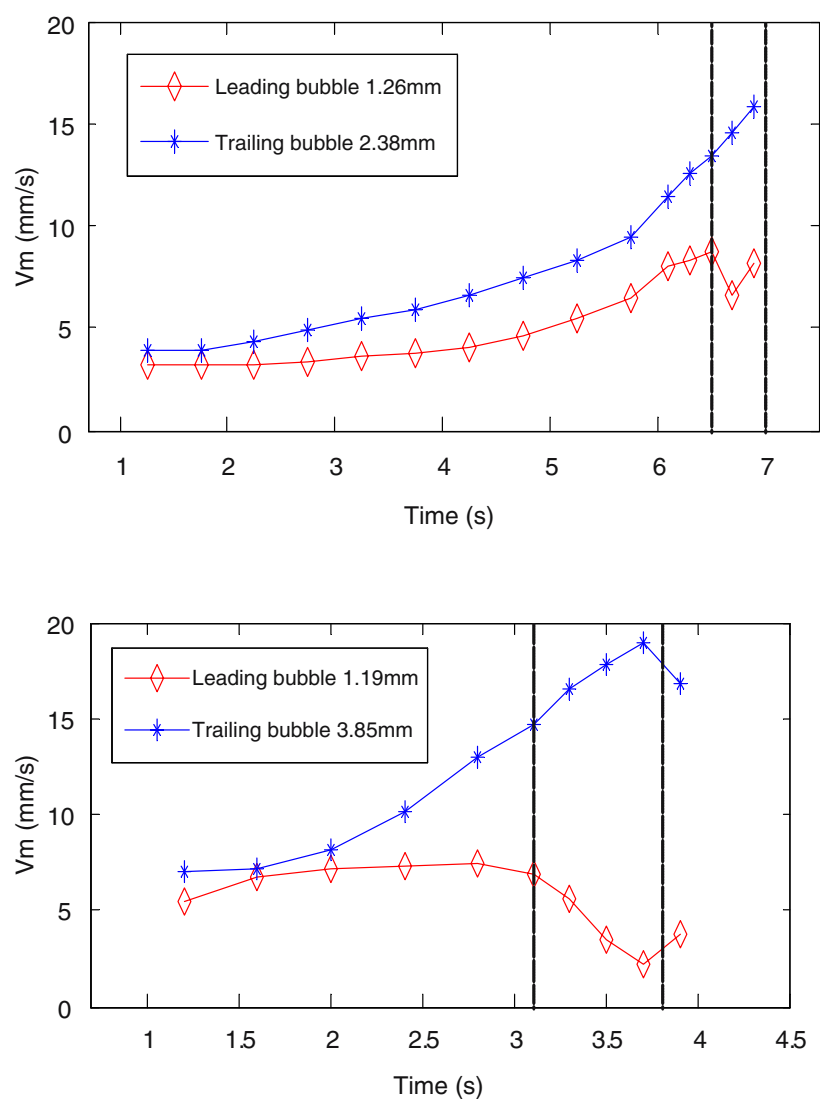

Fig. 4 Location of two bubble and migration velocities versus time. a Radius ratio $r=1.89$. b Radius ratio $r=3.24$ 


\section{Conclusions}

In this space experiment, the maximum $M a$ of bubble thermocapillary migrations reached 9288, which is larger than that attained in any previous space experiments. The migration velocity of bubbles is much smaller than that in the YGB model. The results of the bubble's migration velocity confirmed the trend predicted by Balasubramaniam and Subramanian (1996); Ma et al. (1999) with a wide range of Ma. Experimental results indicated similarities and differences between the experimental results and theoretical analyses. The trajectory and the velocity during the approach were obtained in different ratios of bubble size. The obvious attenuation of the small bubble's velocity due to the interaction between bubbles was displayed in this experiment, which is consistent with Sun and Hu's theoretical results (Sun and $\mathrm{Hu}$ 2002, 2003). Furthermore, the interaction range of two bubbles was also presented clearly.

Acknowledgments This work was supported by the National Nature Science Foundation of China $(10432060,10672171)$ and Knowledge Innovation Program of Chinese Academy of Sciences (KJCX2-SW-L05).

\section{References}

Anderson, J.L.: Droplet interactions in thermocapillary motion. Int. J. Multiph. Flow 11, 813-824 (1985)

Balasubramaniam, R., Subramanian, R.S.: Thermocapillary bubble migration-thermal boundary layers for large Marangoni numbers. Int. J. Multiph. Flow 22, 593-612 (1996)
Balasubramaniam, R., Lacy, C.E., Wozniak, G., Subramanian, R.S.: Thermocapillary migration of bubbles and drops at moderate values of the Marangoni number in reduced gravity. Phys. Fluids 8, 872-880 (1996)

Hadland, P.H., Balasubramaniam, R., Wozniak, G., Subramanian, R.S.: Thermocapillary migration of bubbles and drops at moderate to large Marangoni number and moderate Reynolds number in reduced gravity. Exp. Fluids 26, 240-248 (1999)

Ma, X., Balasubramaniam, R., Subramanian, R.S.: Numerical simulation of thermocapillary drop motion with internal circulation. Numerical Heat Transfer Part A 35, 291-309 (1999)

Meyyappan, M., Subramanian, R.S.: The thermocapillary motion of two bubbles oriented arbitrarily relative to a thermal gradient. J. Colloid Interface Sci. 97, 291-294 (1984)

Meyyappan, M., Wilcox, W.R., Subramanian, R.S.: The slow axisymmetric motion of two bubbles in a thermal gradient. J. Colloid Interface Sci. 94, 243-257 (1983)

Nas, S., Tryggvason, G.: Thermocapillary interaction of two bubbles or drops. Int. J. Multiph. Flow 29, 1117-1135 (2003)

Nas, S., Muradoglu, M., Tryggvason, G.: Pattern formation of drops in thermocapillary migration. Int. J. Heat Mass Transfer 2006, in press.

Subramanian, R.S., Balasubramaniam, R.: The motion of bubbles and drops in reduced gravity. Cambridge University Press, London (2001)

Subramanian, R.S., Balasubramaniam, R., Wozniak, G.: Fluid mechanics of bubbles and drops. In: Physics of fluids in microgravity. Gordon \& Breach, Amsterdam, 2001. 149-177

Sun, R., Hu, W.R.: The thermocapillary migration of two bubbles in microgravity environment. J. Colloid Interface Sci. 255, 375-381 (2002)

Sun, R., Hu, W.R.: Planar thermocapillary migration of two bubbles in microgravity environment. Phys. Fluids A15, (10), 3015-3027 (2003)

Xie, J.C., Lin, H., Zhang, P., Liu, F., Hu, W.R.: Experimental investigation on thermocapillary drop migration at large Marangoni number in reduced gravity. J Colloid Interface Sci. 285, (2), 737-743 (2005)

Young, N.O., Goldstein, J.S., Block, M.J.: The motion of bubbles in a vertical temperature gradient. J. Fluid Mech. 11, 350-356 (1959) 